\title{
Relação entre Sinal/Sintoma e Tempo para Resolução do Problema de Pacientes em Unidade de Emergência
}

\section{Relationship between Sign/Symptom and Time for Problem Solving of patients Admitted to an Emergency Unit}

\author{
KEILA MARIA DE AZEVEDO PONTE ${ }^{1}$ \\ MARIASINARAFARIAS ${ }^{2}$ \\ MARIA GABRIELA MIRANDA FONTENELE ${ }^{3}$ \\ FRANCISCO ELINALDO SANTIAGO BASTOS ${ }^{4}$ \\ JOSÉ GARCIA DE SOUSA ${ }^{5}$
}

\section{RESUMO}

Objetivo: Verificar a relação entre sinal/sintoma e o tempo para resolução do problema de pacientes em unidade de emergência. Material e Métodos: Pesquisa qualitativa realizada de novembro de 2014 a janeiro de 2015 com 85 pacientes hospitalizados na Emergência do Hospital Santa Casa de Misericórdia de Sobral-Ceará. Os dados foram coletados por meio de uma entrevista individual e analisados a partir da Análise de Conteúdo de Minayo. Resultados: O início dos sinais/sintomas variou, desde a ocorrência no dia da hospitalização até queixas crônicas há oito meses. Quanto ao tempo entre o aparecimento dos sinais/sintomas até a chegada ao hospital, variou de meia hora até seis dias. Já o tempo da admissão no hospital à efetivação do tratamento, os relatos foram de satisfação quanto ao tempo rápido, apesar de alguns descontentamentos com a demora percebida. Conclusão: Em se tratando de cuidados em serviços de emergência, o tempo entre o início dos sinais e sintomas e a efetivação do tratamento deve ser considerado em todos os atendimentos, visto que o problema de saúde deve ser solucionado com maior brevidade possível com vistas a prevenir complicações.

\section{DESCRITORES}

Emergências -Sinais e Sintomas -Tempo.

\begin{abstract}
Objective: To investigate the relationship between sign/ symptom and the time for problem solving of patients admittedto an emergency unit. Materials and Methods: This was aqualitative study carried out from November 2014 to January 2015 with 85 patients admitted to the Emergency Department of Santa Casa de Misericórdia Hospital in Sobral, Ceará. The data werecollected through individual interviews and analyzed using Minayo's Content Analysis. Results: The onset of signs/symptoms reported by patients varied from the day of hospitalization to chronic complaints dating back to eight months. The time between the onsetof signs/ symptoms until the patient's arrival at the hospital ranged from half an hour to six days. However, the time for patient admission to effective carewas reported to be fast, despite some dissatisfaction was also noted by participants. Conclusion: As for care in emergency services, the time between the onset of signs and symptoms and the completion of treatment should be considered in all cases, since the health issueis tobe resolved timelyso that to prevent further complications.
\end{abstract}

\section{DESCRIPTORS}

Emergency-Signals and symptoms-Time.

1 Docente do Curso de Graduação em Enfermagem do Instituto Superior de Teologia Aplicada (INTA). Pesquisadora do Grupo de Pesquisa Promoção e Educação em Saúde de Pessoas em Estado de Vulnerabilidade (GRUPPESPEV). Diretora adjunta de Ensino, Pesquisa e Extensão da Santa Casa de Misericórdia (SCMS) de Sobral. Ceará.Brasil.

2 Acadêmica do Curso de Graduação de Enfermagem das Faculdades INTA em Sobral-CE. Membro do (GRUPPESPEV). Bolsista da Santa Casa de Misericórdia de Sobral. Ceará. Brasil.

3 Acadêmica do Curso de Enfermagem das Faculdades INTA. Bolsista da Fundação Cearense de Apoio à Pesquisa (FUNCAP). Membro do Grupo de Pesquisa e Extensão Cuidadores do Coração do Curso de Enfermagem do INTA. Sobral. Ceará. Brasil.

4 Preceptor do Curso de Enfermagem das Faculdades INTA em Sobral. Ceará. Brasil.

5 Farmacêutico e Diretor financeiro da Santa Casa de Misericórdia de Sobral. Ceará. Brasil. 
A o adoecer a pessoa se torna vulnerável fisicamente e psicologicamente, necessitando de cuidado aliado à atenção e agilidade para a resolução do problema que está ocasionando tal fragilidade, assim, é uma responsabilidade dos profissionais que atuam no setor juntamente com a gestão do serviço proporcionar um atendimento rápido e eficaz para a resolução de problemas de saúde.

O setor de urgência e emergência de um hospital, é destinado a realizar procedimentos para aliviar imediatamente situações bem restritas, e não se destina a incluir um longo plano de cuidados. Assim, os pacientes procuram o serviço de emergência para obter uma atenção imediata, que alivie os sinais e sintomas apresentados ${ }^{1}$.

Deste modo, há um aumento da demanda de emergência o que gera um tipo de cuidado que não consegue criar um vínculo com o serviço de saúde ou de ligação com cuidado em curso, a fim de prevenir complicações e novas doenças. Essa utilização de serviços de emergência é, assim, considerada inadequada ${ }^{1}$.

O uso inadequado de serviços de emergência é um problema comum em vários países. No Brasil, parte expressiva da população busca consultas nos pronto-socorros dos hospitais, mesmo com a ampliação da oferta de serviços de AB. Assim, os hospitais ainda são importantes portas de entrada para a assistência à saúde ${ }^{2}$.

A utilização inadequada dos serviços de emergência é prejudicial para os pacientes graves, que precisam de atendimento oportuno, e para os não graves, que, ao buscarem 0 atendimento hospitalar, não têm garantido o seguimento ${ }^{1}$.

Nesse sentido, uma consequência fortemente apresentada com a utilização inadequada dos serviços de emergência, diz respeito ao tempo decorrido entre o aparecimento inicial de sinais e sintomas de casos emergenciais até a efetivação do tratamento necessário, no qual é a causa principal de complicações evitáveis, podendo ocasionar ainda o óbito.

No entanto, existem fatores que interferem na demora dos atendimentos. Assim, sabe-se que a superlotação dos serviços de emergência é um dos fatores principais, juntando-se ao número de profissionais insuficiente, falta de recursos materiais aliado à desorganização do serviço ${ }^{3}$.
O tempo é um forte concorrente quando se fala em situações emergenciais, pois quando se trata de emergência, o problema deve ser resolvido imediatamente pois há algo que emerge colocando a pessoa em risco iminente de morte ${ }^{4}$.

Com isso, ao ser criado o Acolhimento com Classificação de Risco, que contribui para garantir o acesso do paciente, diminuir o tempo de espera, diminuir o risco e ocorrências iatrogênicas e melhorar a qualidade do atendimento, destaca em cada situação um tempo limite para sua solução, esse tempo significa uma margem provável de segurança, onde o quadro não evoluirá com rapidez, no qual o tempo será suficiente para seu atendimento ${ }^{5}$.

Após o atendimento inicial, procedimentos devem ser realizados para a problemática, assim necessita-se da união da equipe para as atividades serem desenvolvidas com êxito.

Desse modo, ao observar a insatisfação dos usuários com os serviços prestados pela Emergência do segundo maior hospital do Estado do Ceará, percebeu-se que existe a necessidade de conhecer de forma real o tempo que decorre entre o aparecimento inicial dos sinais e sintomas até a efetivação do tratamento, afim de discutir melhorias para essa problemática. Assim, analisar essa temática, é relevante por contribuir nas respostas aos questionamentos mais comuns e com isso contribuir para a melhoria dos serviços de urgência e emergência do referido hospital. Deste modo, objetivamos com esse trabalho verificar a relação entre sinal/sintoma e o tempo para resolução do problema de pacientes em unidade de emergência.

\section{MATERIAL E MÉTODOS}

Trata-se de uma pesquisa qualitativa com vistas a aprender a realidade concreta, permitindo a compreensão dos processos de trabalho, bem como as relações estabelecidas entre as equipes que atuam na Unidade de Urgência e Emergência da Santa Casa de Sobral e sua relação com os pacientes por elas atendidos, sendo assim será mais importante uma compreensão mais abrangente dos dados e não a generalização dos mesmos $^{6}$.

As informações foram coletadas de novembro 
de 2014 a janeiro de 2015, com 85 pacientes atendidos no Serviço de Emergência da Santa Casa de Misericórdia de Sobral. Este que é um hospital especializado em atendimento de média a alta complexidade, sendo referência para Zona Norte do Estado do Ceará.

A escolha dos participantes foi realizada por sorteio entre os presentes na unidade no período da coleta. Como critérios de inclusão: maior de 18 anos, pacientes que estivessem internados na média complexidade, com tempo de admissão no serviço de 12 a 24 horas.Como critério de exclusão: pacientes emestado geral grave, com nível de consciência alterado, com dor de moderada a grave ou alguma dificuldade na fala que dificulte o entendimento pelos pesquisadores.

Participaram da pesquisa 85 pacientes internados no setor da Emergência, os quais foram convidados a participar da pesquisa, momento em que foram explicados os objetivos e os aspectos éticos da pesquisa com seres humanos, com a assinatura do Termo de Consentimento Livre e Esclarecido (TCLE). Após foi realizado uma entrevista por meio de um instrumento de coleta de dados com perguntas abertas e fechadas, contendo questões sobre o perfil sociodemográfico e clínico, sinais e sintomas, tempo para efetivação do tratamento, atuação da equipe multidisciplinar para o paciente, medicamentos em uso, situações de conforto e desconforto.

A análise das informações foi realizada a partir da Análise de Conteúdo de Minayo. Foram respeitados todos os aspectos éticos da pesquisa envolvendo seres humanos, como confidencialidade e privacidade dos indivíduos pesquisados garantindo a proteção da imagem dos mesmos.

Assim, esta pesquisa está vinculada ao projeto "Cuidando Para Prevenir o Adoecimento Cardiovascular" aprovado pelo Comitê de Ética e Pesquisa da Universidade Estadual Vale do Acaraú, obtendo como número 793.626. Para respeitar o anonimato, os participantes foram identificados com a sigla PHE (paciente hospitalizado na emergência), seguido do número sequencial da realização da entrevista (ex. PHE10).

\section{RESULTADOS E DISCUSSÃO}

A idade dos participantes variou de 19 a 75 anos de idade, sendo 16 partícipes com idade entre
19-30, 24 com idade entre 31- 40 anos, 29 com idade de 41-50, oito entre 51-60 anos de idade e oito com idade de 61-75 anos. O estado civil diversificou entre solteiros (29), casados (53), viúvos (1) e divorciados (2). Quanto à escolaridade, haviam os analfabetos (12), os alfabetizados (8), os com ensino fundamental completo (15) e incompleto (26) e com ensino médio completo (13), ensino médio incompleto (4), ensino superior completo (5), ensino superior em andamento (2). Os mesmos tinham procedência de hospitais de toda a região norte que tem com referência para atendimento de alta complexidade foram hospitalizados na Santa Casa de Misericórdia de Sobral-Ceará.

Ao serem questionados no momento da entrevista acerca do horário de início dos sinais/ sintomas que culminaram a sua ida ao hospital, houve relatos de sintomas cronificados (oito meses) até sintomas mais agudos com ocorrência no dia. "A primeira vez que senti foi há oito meses atrás e parou de repente, aí estava no trabalho e deu"PHE10

"Foi lá pra segunda - feira passada a
noite"PHE03. "Mais de uma semana
já"PHE31
"Hoje, às quatro e meia da
manhã"PHE76

Os sinais e sintomas configuram como elemento indispensável na compreensão de problemáticas, assim, diferentes abordagens clínicas são realizadas de acordo com o que for relatado pelo paciente, bem como pelo que é percebido pelo profissional de saúde. Sendo de extrema importância a agilidade da realização de cuidados logo após o aparecimento destes ${ }^{7}$.

No Brasil, existem hospitais de referência em Urgência e Emergência que funcionam como porta de livre acesso para os pacientes, independentemente da sua gravidade estando vulnerável a situações imprevisíveis e a um fluxo contínuo de pessoas ${ }^{8}$. Assim, servem de referência para a macrorregião onde se localizam. O hospital Santa Casa de Misericórdia de Sobral, é a referência para a região Norte do Estado do Ceará.

Nesse sentido, ao ser questionado sobre a realização de encaminhamentos por serviços de saúde para o serviço hospitalar emergencial, os 
relatos mostraram que alguns serviços não realizaram encaminhamentos, enquanto outros realizaram de acordo com a necessidade apresentada, de forma efetiva e outros demonstraram insatisfação relacionada a encaminhamentos tidos como desnecessários a outras unidades hospitalares devido à indefinição do diagnóstico apresentado pelo paciente.

"Não, vim direto pra cá, o SAMU me trouxe"PHE65

"Estive no posto de saúde do meu distrito pra fazer o encaminhamento"PHE83

"Sim, na Unidade mista e me encaminharam pra cá"PHE48

"Sim, procurei o hospital de minha cidade aí eles me encaminharam pra outro hospital, lá passei 03 dias internado, aí voltei pra casa, depois de 03 dias piorei de novo e me encaminharam pra cá"PHE36 "Primeiro eu passei no Pronto Socorro lá de Forquilha, depois me botaram no Hospital do Coração pra fazer um eletro e do hospital do Coração me mandaram pra outro hospital onde fiquei a noite todinha internada e depois me mandaram pra cá"PHE72

Os resultados mostram que os encaminhamentos ocorreram de Estratégias de Saúde da Família (ESF), Serviço de Atendimento Móvel de Urgência (SAMU) e de outros hospitais. Deste modo, é necessário que o acesso ao serviço de emergência, seja condicionado por encaminhamentos do local de origem de acordo com a necessidade apresentada, sendo assim necessário conhecimento para tal habilidade ${ }^{9}$.

Ao ser perguntado de uma forma geral o tempo decorrido entre o aparecimento dos sinais/ sintomas até a chegada ao hospital Santa Casa, alguns dos participantes contaram que o tempo foi longo, outros relataram ter sido rápido, sem demora.

\section{"Seis dias"PHE15}

"Não, não, demorou muito não, foi ligeiro. Uma meia hora, porque teve que esperar o SAMU"PHE03

"Demorou umas duas horas pra mim vim"PHE27
Destaca-se que no quesito tempo de chegada ao serviço existem fatores que são responsáveis pelo aumento do tempo, como por exemplo: a distância do município de origem até o hospital Santa Casa, questões culturais do hábito de não procurar serviços de saúde e ainda a falta de conhecimentos e habilidades profissionais quanto a não realização efetiva de encaminhamentos a especialidades.

Em um estudo realizado em São Paulo, foi identificado que a distância geográfica é um importante obstáculo para o atendimento emergencial efetivo, pois o tempo perdido até a chegada na unidade pode causar sérias complicações ao paciente? .

Atualmente destaca-se que os hábitos culturais é um fator preponderante no processo saúde-doença, pois provoca alterações em conceitos relacionados a doença e a saúde, levando a pessoa a não acreditar em medidas promocionais de saúde ${ }^{10}$.

Contudo, ao ser indagado acerca do tempo decorrido entre a chegada no hospital Santa Casa e a efetivação do tratamento, a etapa final da hospitalização, existiram relatos de satisfação quanto ao tempo de tratamento, pois o atendimento recebido foi rápido, outros demonstraram descontentamento com a demora percebida.
"Assim que eu cheguei logo me atenderam, me deram um soro, assim que cheguei já me deram remédio"PHE13
"Assim que eu cheguei fui logo para a sala de medicação, não sei quanto tempo deu" PHE18
"Demorou, porque eu eu cheguei aqui era 5 horas e eu passando mal, vomitando"PHE17
"Demorou, quase 01 hora, pois fiquei sentado na cadeira"PHE08
"Demorou pra me ser atendida, por que eles estavam atendendo os pacientes mais graves" PHE59

Em situações de emergência, o atendimento da vítima deve ser realizado de forma rápida, objetiva e eficaz, proporcionando aumento da sobrevida e a redução de sequelas ${ }^{11}$.

A demora no atendimento é considerada um 
problema que põe em risco a qualidade do atendimento, isto implica a necessidade de um gerenciamento adequado das práticas realizadas ${ }^{12}$.

No momento da entrevista, ao serem questionados acerca do tempo decorrido desde sua entrada no serviço até o atendimento médico, foi relatado por muitos pacientes que foi um período de tempo efetivo, outros negaram a efetividade.

\section{"Não foi ideal, pois poderia ser mais rápido" PHE65 \\ "Foi ideal sim o atendimento médico" PHE23 \\ "Quando a gente está sentindo dor, nada é ideal, mas está de bom tamanho" PHE10}

$O$ atendimento no serviço de urgência e emergência é considerado ideal quando consegue resolver os problemas apresentados pelos pacientes. À medida que o paciente chega à unidade, determina-se um tempo ideal para seu atendimento, a partir da gravidade do caso. Isto é imprescindível em um serviço de urgência e emergência que lida com a necessidade de cuidado imediato $^{13}$.

Portanto, tratar o paciente em tempo ideal, com qualidade no atendimento, valorizando seus sentimentos e promovendo conforto é capaz de salvar vidas e principalmente, proporcionar um atendimento humanizado e com tratamento eficaz.

\section{CONCLUSÃO}

Os resultados mostraram que o início dos sinais/sintomas variou, desde a ocorrência no dia da hospitalização até queixas cronificadas há oito meses. Os encaminhamentos ocorreram de Estratégias de Saúde da Família (ESF), Serviço de Atendimento Móvel de Urgência (SAMU) e de outros hospitais, no entanto houve relatos em que não houve encaminhamentos, tendo o paciente ido por conta própria.

Quanto ao tempo decorrido entre o aparecimento dos sinais/sintomas até a chegada ao hospital variou de meia hora até seis dias. Contudo, da admissão no hospital à efetivação do tratamento, os relatos foram de satisfação quanto ao tempo rápido, apesar de alguns descontentamentos com a demora percebida.

Em se tratando de cuidados em serviços de emergência, o tempo entre o inicio dos sinais e sintomas e a efetivação do tratamento deve ser considerado em todos os atendimentos, visto que o problema de saúde deve ser solucionado com maior brevidade possível com vistas a prevenir complicações.

Deste modo, torna-se relevante promover estratégias para melhorar a qualidade do atendimento nos serviços de emergência de hospitais públicos brasileiros, iniciando com medidas gerenciais organizacionais, com vistas a diminuir o tempo de espera para atendimentos, revendo a forma de condução dos métodos de trabalho, bem como a ampliação do espaço físico e mais equipamentos.

Aliado a isto, o acolhimento se torna uma ação tecno-assistencial que pressupõe a mudança da relação profissional-usuário através de parâmetros técnicos, éticos, humanitários e de solidariedade. E o Acolhimento com Classificação de Risco é uma forma de estratificar o risco de vida do paciente em emergência e consequentemente prover o tempo necessário para seu atendimento.

\section{REFERÊNCIAS}

1. Carret ML, Fassa AC, Domingues MR. Inappropriate use of emergency services: a systematic review of prevalence and associated factors. Cad Saude Publica.2009;25(1):7-28.

2. Machado CV, Salvador FGF, O’dwyer G. Serviço de Atendimento Móvel de Urgência: análise da política brasileira. Physis. 2011; 45(3):519528.

3. Souza LPSS et. al. A qualidade do atendimento prestado pelos prontos-socorros de hospitais públicos do Brasil. Rahis.2014;11(3):205-12.

4. Nascimento KC, Gomes AMT, Erdmann AL. A estrutura representacional do cuidado intensivo para profissionais de Unidade de Terapia Intensiva móvel. Rev Esc de Enferm. USP. 2013; 47(1):176-84. Acesso em: 18 de jul de 2015. 
5. Souza RS, Bastos MAR. Acolhimento com classificação de risco: o processo vivenciado por profissional enfermeiro. Rev MinEnferm. 2008; 12(4): 581-86.

6. Minayo MCS. O desafio do conhecimento: pesquisa qualitativa em saúde.10ª ed. São Paulo: Hucitec, 2007.

7. Souza ACC, Moreira TMM, Borges JWP, Andrade AM, Andrade MM, Almeida PC.Caracterização clínicoepidemiológica da clientela com crise hipertensiva atendida em um serviço de emergência de um hospital municipal de Fortaleza-CE. Rev Min Enferm. 2009;13(1): 13-18.

8. Oliveira JDS, Achieri JC, Pessoa Júnior JM, Miranda FAN, Almeida MG. Representações sociais de enfermeiros acerca do estresse laboral em um serviço de urgência. Rev Esc de Enferm USP. 2013; 47 (4): 984-89.

9. Spedo SM, Pinto NRS, TANAKA OY. O difícil acesso a serviços de média complexidade do SUS: o caso da cidade de São Paulo, Brasil. Physis. 2010;20(3): 95372.

10. Felipe GF, Educação em saúde em grupo: olhar da enfermeira e do usuário hipertenso,[Dissertação de Mestrado]. Fortaleza: Universidade Estadual do Ceará. 2011. 173p.
11. Pergola AM, Araújo IEM. O leigo em situação de emergência. Rev Esc Enferm. 2008; 42(4): 769-76.

12. Andrade LM, Martins EC, Caetano JA, Soares E, Beserra EP.Atendimento humanizado nos serviços de emergência hospitalar na percepção do acompanhante. Rev Eletrônica Enferm. 2009;11(1):151-7.

13. Guedes HM, Valor de predição do protocolo de Manchester em um hospital do Vale Jequitinhonha, [Tese de Doutorado]. Minas Gerais: Universidade Federal de Minas Gerais 2014. 107p.

\section{Correspondência}

Maria Sinara Farias

Rua Eurípedes Ferreira Gomes, № 341, Apartamento 01. Pedrinhas - Sobral - Ceará - Brasil.

CEP: $62040-750$

E-mail: sinarafariasbc@gmail.com 